Pacific Journal of Mathematic 


\title{
IMAGES OF ORDERED COMPACTA ARE LOCALLY PERIPHERALLY METRIC
}

\section{SIBE MARdeší́}

\begin{abstract}
In this paper we study the class $\Omega$ of Hausdorff compact spaces $X$ which are obtainable as images of ordered compacta $K$ under (continuous) maps $f: K \rightarrow X$ onto $X$. The topology of $K$ is the order topology induced by a total (linear) ordering $<$ on $K$. We find that $X$ is locally peripherally metric (Theorem 5), i.e., it has a basis of open sets with metrizable frontiers.
\end{abstract}

In fact, our main result is this stronger statement.

THEOREM 1. Let $X$ be a continuous image of an ordered compactum $K$ and let $G$ be an open $F_{o}$-set in $X$. If $\mathrm{Cl} G$ is connected, then the frontier $\mathrm{Fr} G$ is metrizable.

Theorems 1 and 5 answer in the affirmative two questions raised by the author in [3].

As an immediate consequence, we obtain

Corollary 1. Let $X$ be a continuous image of an ordered compactum $K$ and let $G$ be an open $F_{o}$-set in $X$. If every point $x \in \mathrm{Fr}$ $G$ has a connected open neighborhood in $\mathrm{Cl} G$, then $\mathrm{Fr} G$ is metrizable.

Another easy consequence of Theorem 1 is the following theorem of L. B. Treybig [10]:

CoRollaRY 2 (Treybig). Let $X$ be a continuous image of an ordered compactum $K$. If $X$ is connected and separable, then it is metrizable.

The proof of Theorem 1 given in $\$ 5$ depends on an apparently new metrization theorem for Hausdorff compact spaces (Theorem 2 of $\S 1$ ), on earlier work of the author on separation properties of images of ordered compacta [3], on the earlier joint work with P. Papic ([5], [6],) and on the following product theorem due to A. J. Ward [13] and L. B. Treybig [9] (see also [3] and [4]).

Product Theorem (Ward, Treybig). Let $X$ and $Y$ be infinite compacta such that $X \times Y$ is the image of an ordered compactum. Then both $X$ and $Y$ are metrizable.

The proof of Theorem 1 does not depend on Corollary 2 and, 
therefore provides a new proof of this important result (For another proof of Corollary 2 see [3]).

1. A metrization theorem for Hausdorff compacta. In this paper a compactum is a Hausdorff compact space and a continuum is a connected compactum. If $Y$ is a compactum, $Z(Y)$ denotes the space of components of $Y$, i.e., $Z(Y)$ is the quotient space $Y / R$, where $y R y^{\prime}, y, y^{\prime} \in Y$, means that both $y$ and $y^{\prime}$ belong to the same (connected) component of $Y$. It is well-known that $Z(Y)$ is again a compactum and that the natural projection $\pi: Y \rightarrow Z(Y)(\pi(y)$ is the component of $y$ in $Y$ ) is a continuous mapping onto (see e.g. [8]).

We now consider, for compacta $Y$, the following two properties:

Property $\mu$. For every closed subset $A \subset Y$ the space of components $Z(A)$ is metrizable.

Property $\sigma$. There exists a countable family $\subseteq$ of open sets $S$ such that for any pair of disjoint closed sets $M, N \subset Y$ there exists an $S \in \mathbb{S}$ which separates $Y$ between $M$ and $N$.

We say that $S$ separates $Y$ between $M$ and $N$ provided there exist disjoint sets $A, B \subset Y$ such that $M \subset A, N \subset B, A \cup B=Y \backslash S$, and $A$ and $B$ are both closed in $A \cup B$.

THEOREM 2. In order that a compactum $Y$ be metrizable it is necessary and sufficient that it has both properties $\mu$ and $\sigma$.

Proof. If $Y$ is metrizable, then so are its closed subsets $A \subset Y$. Therefore, their continuous images $Z(A)=\pi(A)$ are also metrizable, so that $Y$ has property $\mu$.

To prove that $Y$ has property $\sigma$, consider a countable basis $\mathfrak{S}$ which is closed under finite unions. Given any pair of disjoined closed sets $M, N \subset Y$, one readily finds a closed set $F \subset Y \backslash(M \cup N)$ which separates $Y$ between $M$ and $N$. Now it suffices to cover $F$ by a set $S \in \mathcal{S}$ which does not meet $M \cup N$.

Suppose now that $Y$ is a compactum with properties $\mu$ and $\sigma$. We construct a countable basis $\mathfrak{B}$ for the topology of $Y$ as follows. Choose, by property $\sigma$, a countable family $\subseteq$ and consider for each $S \in \subseteq$ the closed set $Y \backslash S$. Next, choose a countable basis $\mathscr{B}_{S}^{*}$ for the topology of the metric compactum $Z(Y \backslash S)$ (property $\mu$ ). Let $\mathcal{B}_{S}$ consist of all sets of the form

$$
U=S \cup \pi^{-1}(V),
$$

where $V \in \mathscr{B}_{S}^{*}$ and $\pi: Y \backslash S \rightarrow Z(Y \backslash S)$ is the natural projection. Clearly 


$$
\mathfrak{B}=\cup \mathfrak{B}_{S}, S \in \mathfrak{S},
$$

is a countable collection of open sets of $Y$.

To show that $\mathfrak{B}$ is a basis for $Y$, consider a point $y_{0} \in Y$ and a closed set $M \subset Y, y_{0} \notin M$. We shall exhibit a set $U \in \mathfrak{B}$ such that $y_{0} \in U$ and $U \cap M=0$.

First take an open set $S_{0} \in \mathfrak{S}$ which separates $Y$ between $y_{0}$ and $M$. Then choose a decomposition of $Y \backslash S_{0}$ in two disjoint closed sets $A, B$ such that $y_{0} \in A, M \subset B$. No component of $Y \backslash S_{0}$ meets simultaneously $A$ and $B$. Hence,

$$
\pi(A) \cap \pi(B)=0,
$$

where $\pi: Y \backslash S_{0} \rightarrow Z\left(Y \backslash S_{0}\right)$ is the natural projection. We obtain thus a decomposition

$$
Z\left(Y \backslash S_{0}\right)=\pi(A) \cup \pi(B)
$$

of $Z\left(Y \backslash S_{0}\right)$ in two disjoint closed and open subsets $\pi(A), \pi(B)$. Since,

$$
\pi\left(y_{0}\right) \in \pi(A),
$$

there exists an open set $V \in \mathfrak{B}_{S_{0}}^{*}$ such that

$$
\pi\left(y_{0}\right) \in V \subset \pi(A) \text {. }
$$

Consequently,

$$
y_{0} \in \pi^{-1}(V) \subset A
$$

and we see that the set

$$
U=S_{0} \cup \pi^{-1}(V) \in \mathfrak{B}_{S_{0}} \subset \mathfrak{B}
$$

fulfills the requirements

$$
y_{0} \in U, \quad U \cap M=0 .
$$

This completes the proof of Theorem 2 .

REMARK. Property $\sigma$ alone is not sufficient to imply metrizability of $Y$. E.g. every separable ordered compactum $K$ has property $\sigma$ (see Theorem 4 in $\S 3$ ), but $K$ need not be metrizable. The corresponding question for property $\mu$ is discussed in $\$ 2$.

2. Property $\mu$ and the Suslin problem. ${ }^{1}$ A space $Y$ is said to have the Suslin property if every family of nonempty disjoint open sets in $Y$ is countable.

\footnotetext{
1 The results of this section are not used in the sections that follow.
} 
LEMMA 1. If a compactum $Y$ has property $\mu$, it also has the Suslin property.

Proof. Let $U=\left\{U_{\lambda}\right\}, \lambda \in L$, be a family of nonempty disjoint open sets in $Y$. Choose, for each $\lambda \in L$, a point $y_{\lambda} \in U_{\lambda}$. Let

$$
A=\mathrm{Cl}\left[\bigcup_{\lambda \in L}\left\{y_{\lambda}\right\}\right] \text {. }
$$

Clearly, the points $y_{\lambda}$ are isolated in the set $A$ and, therefore, $\pi\left(y_{\lambda}\right)$ are isolated points in $Z(A)$. Since, $Z(A)$ is a metrizable compactum, it can have only countably many isolated points. This proves that $L$ is countable, i.e., that $Y$ has the Suslin property.

Lemma 2. Let $C$ be an ordered continuum with the Suslin property. Then $C$ has property $\mu$.

Proof. An ordered continuum $C$ is an ordered compactum which is connected. If $A$ is a closed subset of $C$, then the open set $C \backslash A$ decomposes in a countable family of maximal disjoint open intervals $U_{n}$. Clearly, the space of components $Z(A)$ is a totally disconnected ordered compactum whose order is induced by the order $<$ in $C$.

By a gap in an ordered compactum $(K,<)$ we mean a pair of points $c_{1}, c_{2} \in K$, such that the interval $\left(c_{1}, c_{2}\right)_{K}$ is empty. It is readily seen that a totally disconnected ordered compactum $K$ with only countably many gaps is metrizable and is in fact a subset of the Cantor set (see e.g. Lemma 1 of [9]).

Thus, in order to show that $Z(A)$ is metrizable it suffices to show that $Z(A)$ has only countably many gaps. In fact, we can associate with every gap $C_{1}, C_{2}$ of $Z(A)$ the unique interval $U_{n} \subset C$ whose two end-points belong to the components $C_{1}$ and $C_{2}$ of $A$ respectively. In this way we obtain a one-to-one mapping of the set of gaps of $Z(A)$ into the set of intervals $U_{n}$. This proves that $Z(A)$ has only countably many gaps and is, therefore, metrizable. Since $Z(A)$ is metrizable, for every closed set $A \subset C$, the continuum $C$ has property $\mu$.

The author does not know of any example of a compactum $Y$ which has property $\mu$ but fails to be metrizable. However, if property $\mu$ alone would imply metrizability of compacta $Y$, then Lemma 2 would imply that every ordered continuum $C$ with the Suslin property is metrizable and, therefore, homeomorphic to the real line segment I. In other words, we would have a positive answer to the Suslin problem (M. Ya. Suslin in Fund. Math. 1 (1920), p. 223).

THEOREM 3. The following two statements are equivalent:

(i) In the class $\Re$ of images of ordered compacta every compactum 
$X \in \Re$ with property $\mu$ is metrizable,

(ii) Every ordered continuum $C$ with the Suslin property is homeomorphic to the real line segment $I$.

Proof. (i) $\Rightarrow$ (ii) is an immediate consequence of Lemma 2.

In order to prove that (ii) $\Rightarrow$ (i), consider a compactum $X \in \Re$ which has property $\mu$. It follows from Lemma 1 that $X$ has the Suslin property. Using (ii), P. Papic and the author have proved that a compactum $X \in \Re$ with the Suslin property is separable (Corollary 6 of [6]), and in $\S 3$ of this paper we prove that every separable compactum $X \in \Re$ has property $\sigma$ (Theorem 4). Hence, $X$ has both properties $\mu$ and $\sigma$ and is therefore metrizable, by Theorem 2 .

3. Images of ordered compacta and property $\sigma$. In this section we prove

THEOREM 4. Let $X$ be a continuous image of an ordered compactum. If $X$ is separable, it has property $\sigma$.

We first recall that if $X \in \Re$ has the Suslin property, then every open subset of $X$ is an $F_{o}$-set (see Theorem 2 of [5] or Corollary 3, p. 13 of [6]). This holds a fortiori if $X$ is separable so that we have

Lemma 3 (Mardešic-Papic). If $X \in \Re$ is separable, then every closed subset of $X$ is a $G_{\delta}$-set and every open subset of $X$ is an $F_{\sigma^{-}}$ set.

Proof of Theorem 4. The author has shown (Theorem 4 in [3]) that a separable $X \in \Re$ admits a countable family $\mathfrak{F}$ of closed sets $F$ which separate $X$ between any pair of disjoint closed sets $M, N \subset X$. We now choose such a family $\mathfrak{F}$.

Each $F \in \widetilde{F}$ is a $G_{\delta}$-set (Lemma 3 ) so that we can choose a countable collection $\widetilde{S}_{F}$ of open sets $S \subset X$ such that $F \subset S$ and

$$
F=\cap(\mathrm{Cl} S), \quad S \in \subseteq_{F} .
$$

The family

$$
\mathfrak{S}=\cup \mathfrak{S}_{F}, \quad F \in \mathfrak{F},
$$

is a countable collection of open sets in $X$ which has the required separation property $\sigma$.

Indeed, if $M$ and $N$ are disjoint closed subsets of $X$, then there exists a set $F \in \mathfrak{F}$ such that $F$ separates $X$ between $M$ and $N$. Since

$$
F \subset X \backslash(M \cup N),
$$


and (1) holds, we can find a set $S \in \mathfrak{S}_{F} \subset \subseteq$ such that

$$
F \subset S \subset \mathrm{Cl} S \subset X \backslash(M \cup N) .
$$

Clearly, such a set $S \in \subseteq$ separates $X$ between $M$ and $N$, which concludes the proof.

4. The frontier of open $\mathrm{F}_{\sigma}$-sets and its space of components.

In this section we prove the crucial

Lemma 4. Let $X \in \Re$ and let $G$ be an open $F_{\sigma}$-set dense in $X$. If $X$ is connected, the space of components $Z(\operatorname{Fr} G)$ is metrizable.

Proof. Choose a sequence of open sets $H_{n} \subset G, n=1,2, \cdots$, such that

$$
\begin{aligned}
& \mathrm{Cl} H_{n} \subset H_{n+1}, \\
& \bigcup_{n=1}^{\infty} \mathrm{Cl} H_{n}=G .
\end{aligned}
$$

For each $n$, consider the compactum

$$
X \backslash H_{n} \supset X \backslash G .
$$

Let

$$
Z_{n}=Z\left(X \backslash H_{n}\right), \quad Z=Z(X \backslash G) .
$$

By (3), every component of $X \backslash G$ is contained in a unique component of $X \backslash H_{n}$. This inclusion defines a map

$$
p_{n}: Z \rightarrow Z_{n} \text {. }
$$

We shall now show that the maps $p_{n}, n=1,2, \cdots$, distinguish points of $Z$, i.e. that for any two distinct components $C_{1}, C_{2}$ of $X \backslash G$ there exists an $n$ such that

$$
p_{n}\left(C_{1}\right) \neq p_{n}\left(C_{2}\right) .
$$

The maps $p_{n}, n=1,2, \cdots$, will thus define an imbedding of $Z$ in the direct product

$$
\prod_{n=1}^{\infty} p_{n}(Z) \text {. }
$$

We first choose two disjoint closed sets $F_{1}, F_{2}$ in $\operatorname{Fr} G$ covering Fr $G$ and such that $C_{1} \subset F_{1}$ and $C_{2} \subset F_{2}$. Since the sets $F_{i}$ are at the same time closed in $X$, we can surround them by disjoint open sets $U_{1}, U_{2}$ of $X$. Thus 


$$
\begin{gathered}
C_{i} \subset U_{i}, \quad i=1,2, \\
U_{1} \cup U_{2} \supset \operatorname{Fr} G .
\end{gathered}
$$

We now choose an $n$ such that

$$
X \backslash\left(U_{1} \cup U_{2}\right) \subset H_{n} .
$$

The set $X \backslash H_{n} \subset U_{1} \cup U_{2}$ splits in two disjoint open sets $U_{i} \cap\left(X \backslash H_{n}\right)$, $i=1,2$, which contain $C_{1}$ and $C_{2}$ respectively. This proves that $C_{1}$ and $C_{2}$ are included in different components of $X \backslash H_{n}$ so that (6) takes place.

In order to complete the proof of Lemma 4 it now suffices to show that the space $p_{n}(Z)$ is metrizable, for every $n$. In that case the direct product (7) will be metrizable and so will be $Z$ itself, because $Z$ is embeddable in this product.

To show that $p_{n}(Z)$ is metrizable, first notice that every component $C$ of $X \backslash H_{n}$ meets $\mathrm{Cl} H_{n}$, because $X$ is connected and compact. Moreover, if $C \in p_{n}(Z)$. Then $C$ also meets $\operatorname{Fr} G$.

Next, consider the natural projection

$$
\pi: X \backslash H_{n} \rightarrow Z\left(X \backslash H_{n}\right)=Z_{n}
$$

and a map

$$
\varphi: X \backslash H_{n} \rightarrow I=[0,1]
$$

such that

$$
\begin{gathered}
\varphi\left(\left(X \backslash H_{n}\right) \cap \mathrm{Cl} H_{n}\right)=0, \\
\varphi(\operatorname{Fr} G)=1 ;
\end{gathered}
$$

$\varphi$ exists by Urysohn's lemma.

Using $\pi$ and $\varphi$ we define the map

$$
\psi=\pi \times \varphi: X \backslash H_{n} \rightarrow Z_{n} \times I .
$$

We now show that

$$
p_{n}(Z) \times I \subset \psi\left(X \backslash H_{n}\right) .
$$

Indeed, if $C \in p_{n}(Z)$, then $C$ meets Fr $G$ and $\mathrm{Cl} H_{n}$ and so $\psi(C)$ meets both $C \times 1$ and $C \times 0$. Since, $\psi(C) \subset C \times I$ and $\psi(C)$ is connected, it follows that

$$
C \times I=\psi(C) \subset \psi\left(X \backslash H_{n}\right)
$$

and (16) is established.

Since $X$ belongs to $\Omega$, we conclude that also $X \backslash H_{n}, \psi\left(X \backslash H_{n}\right)$ and $p_{n}(Z) \times \mathrm{I}$ belong to $\Re$. Therefore, by the product theorem (see the 
introduction) $p_{n}(Z)$ is metrizable. This completes the proof of Lemma 4 .

5. Proof of Theorem 1. We first prove

Lemma 5. Let $X \in \Re$ and let $G$ be an open $F_{\sigma}$-set in $X$. If $\mathrm{Cl}$ $G$ is connected, then $\operatorname{Fr} G$ has property $\mu$.

Proof. Let $A$ be a closed subset of $\operatorname{Fr} G$ and let

$$
\Gamma=(\mathrm{Cl} G) \backslash A \text {. }
$$

Clearly, $\Gamma$ is an open set, dense in $\mathrm{Cl} G$, and

$$
\operatorname{Fr} \Gamma=A \text {. }
$$

We now show that $\Gamma$ is an $F_{\sigma}$-set in $\mathrm{Cl} G=\mathrm{Cl} \Gamma$. In the first place, $\operatorname{Fr} G$ is a separable compactum from $\Omega$, for the author has proved that the frontier of an open $F_{\sigma}$-set in a compactum $X \in \Omega$ is always separable (Theorem 2 of [3]). It follows, by Lemma 3, that $(\mathrm{Fr} G) \backslash \mathrm{A}$ is an $F_{\sigma}$-set.

On the other hand, $G$ is by assumption an $F_{o}$-set. Consequently,

$$
\Gamma=(\operatorname{Fr} G \backslash A) \cup G
$$

is also an $F_{\sigma}$-set in $X$.

Applying Lemma 4 to $\mathrm{Cl} G$ and $\Gamma$, and taking into account (2), we see that $Z(A)=Z(\operatorname{Fr} \Gamma)$ is metrizable. This concludes the proof of Lemma 5 .

Proof of Theorem 1. To complete the proof, notice that Fr $G$ is a separable compactum from $\Re$ and, therefore, has property $\sigma$ (Theorem 4). On the other hand, by Lemma $5, \operatorname{Fr} G$ has also property $\mu$. Thus, by Theorem 2, $\operatorname{Fr} G$ is a metrizable compactum.

Proof of Corollary 1. Let $X \in \Re$ and let $G$ be an open $F_{\sigma}$-set in $X$ with the property that there is a finite collection of connected open sets $U_{1}, \cdots, U_{n}$ in $\mathrm{Cl} G$ such that

$$
\operatorname{Fr} G \subset U_{1} \cup \cdots \cup U_{n} \text {. }
$$

Clearly, $\mathrm{Cl} U_{i}$ belongs to $\Omega$ and is connected. On the other hand, $\left(\mathrm{Cl} U_{i}\right) \cap G$ is an open $F_{\sigma}$-set dense in $\mathrm{Cl} U_{i}$, because $U_{i} \subset \mathrm{Cl} G$ implies

$$
U_{i} \subset \mathrm{Cl}\left[U_{i} \cap G\right] \subset \mathrm{Cl}\left[\mathrm{Cl}\left(U_{i}\right) \cap G\right] \subset \mathrm{Cl} U_{i},
$$

so that

$$
\mathrm{Cl}\left[\mathrm{Cl}\left(U_{i}\right) \cap G\right]=\mathrm{Cl} U_{i} \text {. }
$$


It follows from ( 6 ) and Theorem 1 that

$$
\operatorname{Fr}\left[\mathrm{Cl}\left(U_{i}\right) \cap G\right]=\left(\mathrm{Cl} U_{i}\right) \backslash G
$$

is metrizable. Since, by (4), the sets $\left(\mathrm{Cl} U_{i}\right) \backslash G, i=1, \cdots, n$, cover $\operatorname{Fr} G$, we conclude that $\operatorname{Fr} G$ itself is metrizable.

Proof of Corollary 2. Corollary 2 is an immediate consequence of Theorem 1 and this

Lemma 6. If $X \in \Re$ is separable, there exists a compactum $X^{\prime} \in \Re$ and an open $F_{\sigma}$-set $G \subset X^{\prime}$ dense in $X^{\prime}$ and such that $X=\operatorname{Fr} G$. Moreover, if $X$ is connected, so is $X^{\prime}$.

Proof. Let $f: K \rightarrow X$ be a map of an ordered compactum $K$ onto $X$ and let $D=\left\{t_{1}, \cdots, t_{n}, \cdots\right\}$ be a countable subset of $K$ such that $f(D)$ is dense in $X$. Let $K^{\prime}$ be a new ordered compactum obtained from $K$ by replacing each point $t_{n} \in D$ by a copy $I_{n}$ of the real line segment $I$. We denote the two end-points of $I_{n}$ by $t_{n}^{\prime}$ and $t_{n}^{\prime \prime}$ and its interior by $I_{n}^{0}$. $K \backslash D$ can be considered as a subset of $K^{\prime}$.

We now define a map

$$
f^{\prime}: K^{\prime} \rightarrow X \times I
$$

as follows. For $t \in K \backslash D$, let

$$
f^{\prime}(t)=f(t) \times 0,
$$

let

$$
f^{\prime}\left(t_{n}^{\prime}\right)=f^{\prime}\left(t_{n}^{\prime \prime}\right)=f\left(t_{n}\right),
$$

and let $f^{\prime} \mid I_{n}$ be any map of $I_{n}$ onto

$$
f\left(t_{n}\right) \times\left[0, \frac{1}{n}\right]
$$

such that the end-points $t_{n}^{\prime}, t_{n}^{\prime \prime}$ are the only points of $I_{n}$ which are mapped into $f\left(t_{n}\right) \times 0$. It is easy to verify that $f^{\prime}: K^{\prime} \rightarrow X \times I$ is continuous.

We now define $X^{\prime}$ by

$$
X^{\prime}=f^{\prime}\left(K^{\prime}\right) \subset X \times I .
$$

$X^{\prime} \in \Re$ and

$$
X \times 0=f^{\prime}\left(K^{\prime} \backslash \bigcup_{n=1}^{\infty} I_{n}^{0}\right) \subset X^{\prime} .
$$

Clearly, the set 


$$
G=X^{\prime} \backslash(X \times 0)=\bigcup_{n=1}^{\infty} f^{\prime}\left(I_{n}^{0}\right)
$$

is an open $F_{\sigma}$-set in $X^{\prime}$ and

$$
\operatorname{Fr} G=X \times 0,
$$

because $\mathrm{Cl} G \supset f(D) \times 0$ and, therefore,

$$
\mathrm{Cl} G \supset \mathrm{Cl}[f(D) \times 0]=X^{\prime} \text {. }
$$

If $X$ is connected, so is $X^{\prime}$, because it consists of $X \times 0$ and $\operatorname{arcs}(11)$ which meet $X \times 0$.

\section{Local peripheral metrizablity.}

LEMma 7. Let $X$ be a continuous image of an ordered compactum. If $X$ is locally connected, then it is locally peripherally metrizable.

Proof. If $F \subset X$ is a closed connected set and $U \subset X$ is open and $F \subset U$, then one can easily find (using regularity and local connectedness of $X$ ) an open connected set $V$ in $X$ such that

$$
F \subset V \subset \mathrm{Cl} V \subset U \text {. }
$$

Using this argument repeatedly, one can find, for each point $x_{0} \in X$ and each open neighborhood $U$ of $x_{0}$, a sequence of connected open sets $V_{n}, n=1,2, \cdots$, such that

$$
x_{0} \in V_{1} \subset \cdots \subset V_{n} \subset \mathrm{Cl} V_{n} \subset V_{n+1} \subset \cdots \subset U .
$$

Clearly,

$$
\mathrm{V}=\bigcup_{n=1}^{\infty} V_{n}=\bigcup_{n=1}^{\infty} \mathrm{Cl} V_{n}
$$

is a connnected open $F_{\sigma}$-set in $X$ such that

$$
x_{0} \in V \subset U \text {. }
$$

By Theorem 1, Fr $V$ is metrizable, which proves that $X$ is locally peripherally metrizable.

THEOREM 5. Every continuous image $X$ of an ordered compactum $K$ is locally peripherally metrizable.

The result follows immediately from Lemma 7 and this

LEMma 8. Every continuous image $X$ of an ordered compactum $K$ can be embedded in a continuous image $Y$ of an ordered continuum $C$. 
Proof. Insert between any two consecutive points of $K$ a copy of the open real line interval filling thus all the gaps in $K$. Denote the obtained ordered continuum by $C$. Consider $X$ as embedded in a cube $I^{k}$. The map $f: K \rightarrow I^{\kappa}$ can be extended to a continuous map $g: C \rightarrow I^{\kappa}, g \mid K=f$. Clearly, $X \subset Y=g(C)$. Notice that $Y$ is locally connected and thus Lemma 7 applies.

REMARK. Local peripheral metrizability together with local connectedness does not suffice for the conclusion that a compactum $X$ belongs to $\Re$ as the following example shows.

EXAMPLE. Let $\Omega=\left\{\alpha \mid \alpha<\omega_{1}\right\}$ be the set of all countable ordinals. Let $L$ be the ordered continuum obtained by ordering lexicographically the product $\Omega \times[0,1)$ and adjoining a last point $\omega_{1}$. Let $X$ be the quotient space

$$
X=(L \times I) / \omega_{1} \times \mathrm{I} .
$$

$X$ is a nonmetrizable locally connected continuum and is locally peripherally metric. However, $X$ does not belong to $\Omega$, because no two points separate $X$ and every nonmetrizable continuum $X \in \Re$ has such a pair of points (see Theorem 2 of [10]).

The author wishes to express his gratitude to Professor A. J. Ward for many interesting and stimulating conversations concerning the subject of this paper.

\section{BIBLIOGRAPHY}

1. S. Mardešic, Mapping ordered continua onto product spaces, Glasnik Mat.-Fiz. Astronom. 15 (1960), 85-89.

2. - On the Hahn-Mazurkiewicz theorem in nonmetric spaces, Proc. Amer. Math. Soc. 11 (1960), 929-937.

3. - Continuous images of ordered compacta and a new dimension which neglects metric subcontinua, Trans. Amer. Math. Soc. 121 (1966), 424-433.

4. S. Mardešić and P. Papić, Continuous images of ordered continua, Glasnik Mat.Fiz. Astronom. 15 (1960), 171-178.

5. —— Diadičeskie bikompakty $i$ nepreryvnye otobraženija uporjadočennyh bikompaktov, Dokl. Akad. Nauk SSSR 143 (1962), 529-531.

6. - Continuous images of ordered compacta, the Suslin property and diadic compacta, Glasnik Mat.-Fiz. Astronom. 17 (1962), 3-25.

7. - Neki problemi preslikavanja uredenih kompakata (Some problems concerning mappings of ordered compacta), Matematička Biblioteka, Beograd, 25 (1963), 11-22.

8. V. I. Ponomarev, O nepreryvnyh razbienijah bikompaktov, Uspehi Mat. Nauk 12 (1957), 335-340.

9. L. B. Treybig, Concerning continuous images of compact ordered spaces, Proc. Amer. Math. Soc. 15 (1964), 866-871.

10. Concerning continua which are images of compact ordered spaces, Duke Math. J. 32 (1965), 417-422. 
11. A. J. Ward, Notes on general topology II, A generalization of arc-connectedness, Proc. Cambridge Phil. Soc. 61 (1965), 879-880.

12. Notes on general topology III, A non-metric image of an ordered compactum, Proc. Cambridge Phil. Soc. 61 (1965), 881-882.

13. - Some properties of images of ordered compacta with special reference to topological limits (to appear in the Canadian J. Math.)

Received April 18, 1966. During this research the author was visiting the University of Washington on leave from the University of Zagreb.

University of Zagreb, Zagreb, Yugoslavia

University of Washington, Seattle, Washington 


\section{PACIFIC JOURNAL OF MATHEMATICS}

\section{H. ROYDEN}

Stanford University

Stanford, California

\author{
J. P. JANS \\ University of Washington \\ Seattle, Washington 98105
}

\section{EDITORS}

\author{
J. DugundJI \\ Department of Mathematics \\ Rice University \\ Houston, Texas 77001 \\ Richard ARENS \\ University of California \\ Los Angeles, California 90024
}

\section{ASSOCIATE EDITORS}
E. F. BECKENBACH
B. H. NeumanN
F. WOLF
K. YOSIDA

\section{SUPPORTING INSTITUTIONS}

\author{
UNIVERSITY OF BRITISH COLUMBIA \\ CALIFORNIA INSTITUTE OF TECHNOLOGY \\ UNIVERSITY OF CALIFORNIA \\ MONTANA STATE UNIVERSITY \\ UNIVERSITY OF NEVADA \\ NEW MEXICO STATE UNIVERSITY \\ OREGON STATE UNIVERSITY \\ UNIVERSITY OF OREGON \\ OSAKA UNIVERSITY \\ UNIVERSITY OF SOUTHERN CALIFORNIA
}

\author{
STANFORD UNIVERSITY \\ UNIVERSITY OF TOKYO \\ UNIVERSITY OF UTAH \\ WASHINGTON STATE UNIVERSITY \\ UNIVERSITY OF WASHINGTON \\ AMERICAN MATHEMATICAL SOCIETY \\ CHEVRON RESEARCH CORPORATION \\ TRW SYSTEMS \\ NAVAL ORDNANCE TEST STATION
}

Mathematical papers intended for publication in the Pacific Journal of Mathematics should be typewritten (double spaced). The first paragraph or two must be capable of being used separately as a synopsis of the entire paper. It should not contain references to the bibliography. Manuscripts may be sent to any one of the four editors. All other communications to the editors should be addressed to the managing editor, Richard Arens at the University of California, Los Angeles, California 90024.

50 reprints per author of each article are furnished free of charge; additional copies may be obtained at cost in multiples of 50 .

The Pacific Journal of Mathematics is published monthly. Effective with Volume 16 the price per volume (3 numbers) is $\$ 8.00$; single issues, $\$ 3.00$. Special price for current issues to individual faculty members of supporting institutions and to individual members of the American Mathematical Society: $\$ 4.00$ per volume; single issues $\$ 1.50$. Back numbers are available.

Subscriptions, orders for back numbers, and changes of address should be sent to Pacific Journal of Mathematics, 103 Highland Boulevard, Berkeley 8, California.

Printed at Kokusai Bunken Insatsusha (International Academic Printing Co., Ltd.), 7-17, Fujimi 2-chome, Chiyoda-ku, Tokyo, Japan.

PUBLISHED BY PACIFIC JOURNAL OF MATHEMATICS, A NON-PROFIT CORPORATION

The Supporting Institutions listed above contribute to the cost of publication of this Journal, but they are not owners or publishers and have no responsibility for its content or policies. 


\section{Pacific Journal of Mathematics}

A. A. Aucoin, Diophantine systems ............................. 419

Charles Ballantine, Products of positive definite matrices. I ............... 427

David Wilmot Barnette, A necessary condition for d-polyhedrality ............ 435

James Clark Beidleman and Tae Kun Seo, Generalized Frattini subgroups of finite groups ......................................... 441

Carlos Jorge Do Rego Borges, A study of multivalued functions ............. 451

William Edwin Clark, Algebras of global dimension one with a finite ideal

lattice ...............................................

Richard Brian Darst, On a theorem of Nikodym with applications to weak convergence and von Neumann algebras .........................

George Wesley Day, Superatomic Boolean algebras .....................

Lawrence Fearnley, Characterization of the continuous images of all

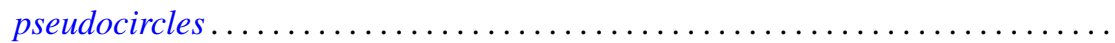

Neil Robert Gray, Unstable points in the hyperspace of connected subsets....... 515

Franklin Haimo, Polynomials in central endomorphisms .................. 521

John Sollion Hsia, Integral equivalence of vectors over local modular lattices . . . . 527

Jim Humphreys, Existence of Levi factors in certain algebraic groups .......... 543

E. Christopher Lance, Automorphisms of postliminal $C^{*}$-algebras ............ 547

Sibe Mardesic, Images of ordered compacta are locally peripherally metric . . . . 557

Albert W. Marshall, David William Walkup and Roger Jean-Baptiste Robert Wets,

Order-preserving functions: Applications to majorization and order

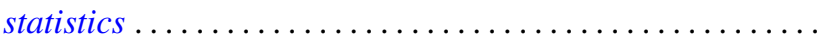

Wellington Ham Ow, An extremal length criterion for the parabolicity of

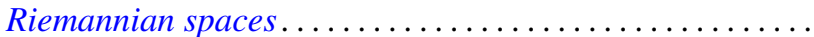

585

Wellington Ham Ow, Criteria for zero capacity of ideal boundary components of

Riemannian spaces...................................... 591

J. H. Reed, Inverse limits of indecomposable continua .................. 597

Joseph Gail Stampfli, Minimal range theorems for operators with thin spectra . . . 601

Roy Westwick, Transformations on tensor spaces..................... 613

Howard Henry Wicke, The regular open continuous images of complete metric

spaces ........................................... 621

Abraham Zaks, A note on semi-primary hereditary rings .............. 627

Thomas William Hungerford, Correction to: "A description of $\operatorname{Mult}_{i}\left(A^{1}, \cdots, A^{n}\right)$

by generators and relations" ............................. 629

Uppuluri V. Ramamohana Rao, Correction to: "On a stronger version of Wallis' formula".............................................. 629

Takesi Isiwata, Correction: "Mappings and spaces" ................... 630

Henry B. Mann, Josephine Mitchell and Lowell Schoenfeld, Correction to:

"Properties of differential forms in $n$ real variables" .... . .

James Calvert, Correction to: "An integral inequality with applications to the

Dirichlet problem"............................. 